\title{
The Status Quo and Future of Traditional and Complementary Medicine (T\&CM) Used During Pregnancy and Postpartum Period among Malaysian Women
}

\author{
Shaukat Ali $\mathbf{R}^{1}$, Gnanasan $\mathbf{S}^{1}$ and Farooqui $\mathbf{M}^{2^{*}}$ \\ ${ }^{1}$ Faculty of Pharmacy, Universiti Teknologi MARA, Puncak Alam Campus, Malaysia \\ ${ }^{2}$ Department of Pharmacy Practice, Unaizah College of Pharmacy, Qassim University, Saudi Arabia \\ *Corresponding author: Maryam Farooqui, Department of Pharmacy Practice, Unaizah College of Pharmacy, Qassim University, Saudi Arabia, Tel: 06-04-5623561; E- \\ mail: maryamfarooqui.uitm@gmail.com
}

Received date: September 26, 2016; Accepted date: September 27, 2016; Published date: September 29, 2016

Copyright: (C) 2016 Shaukat AR, et al. This is an open-access article distributed under the terms of the Creative Commons Attribution License, which permits unrestricted use, distribution and reproduction in any medium, provided the original author and source are credited.

\section{Editorial}

Malaysia is a multi-cultural country situated in Southeast Asia. Since achieving independence, the country has established a modern health care system. However, the diverse culture distribution of its population offers a wide range of traditional ways of healing. These practices reflect on the different cultures and ethnicities in a unique way.

Traditional and complementary medicine (T\&CM) has built up its root in the health care system and an estimated USD 500 million is spent annually on traditional therapies, compared to only USD 300 million on conventional therapies [1].

Traditional Malay medicines are one of the most valuable and rich cultural heritage of Malaysians which reflects on the diverse culture and tradition. While offering the most sophisticated modern health care system to its population, Malaysia also has an integrated medicine program that incorporates selected $\mathrm{T} \& \mathrm{CM}$ practices in ten government hospitals including traditional Malay massage and acupuncture for chronic pain and post stroke management, herbal therapy as an adjunctive treatment for cancer, Malay postnatal care and Shirodhara with the aim to ensure the safety and quality of T\&CM for its consumers [2].

Specific to the use of T\&CM among Malaysian women who are trying to conceive, are pregnant and in the postpartum period, safety of these medications for the developing fetus has not been studied in detail. In general, it is estimated that about 800,000 Malaysian couples are going through sub fertility issues [3].

Furthermore, the perceived high cost of infertility treatment is reported to be a barrier in seeking modern therapies. It has also been reported previously that $\mathrm{T} \& \mathrm{CM}$ are common options among those seeking treatment for infertility, prenatal and postnatal assuming that it is safe and originating from natural sources with affordable prices.

Most of the local products sold are in the form of fertility set, prenatal and postnatal set, juices and supplements. These products are promoted with a vast array of testimonies by people who claim to have used the product on web pages and among blogs. Some women used these local products with modern therapies without prior consultations with a healthcare professional. The increasing influence of traditional cultural practices among childbearing women comes from the popularity of T\&CM in industrialized countries [4].

A survey done in 2006 reported that the most common herbal medicines used during pregnancy were coconut oil, some unidentified herbal medicines acquired from different sources such as aborigines and traditional midwives, local plants known as Kacip Fatimah (Labisia pumila), Manjakani (Croton caudatus), Celaka (Plumbago zeylonica) and the most common indication that these herbal medicines (89.8\%) were used to facilitate labour [5]. Many of the consumers have reported to have used these products without sufficient information on its safety.

Although herbal medicines are always considered to be from natural sources, not all are safe to be used during pregnancy as some of them may have potent active ingredients that might be harmful and it may also stimulate the womb, leading to premature labour, and may cause teratogenicity (congenital anomalies or birth defects). Sometimes, ingredients used might be toxic to the liver and may cause cancer [6].

Although there are thousands of products available in the market for fertility, pregnancy and postpartum care, only few are available with clinical evidence. Whether consumers are trying to conceive, pregnant or in the postpartum care period, consultation from a qualified and registered professional should be sought out before taking any herbal medicines or any other form of T\&CM because there is a high chance of exposure to biologically active compounds.

Therefore, further studies are urgently needed in Malaysia to evaluate the characteristics of pregnant T\&CM users, motivation for T\&CM use, disclosure of T\&CM use to health care providers, T\&CM information sources as well as T\&CM expenditure.

Hence, the outcome of such findings may help to investigate the efficacy and safety of these T\&CM modalities. It will also be a valuable bench mark in evaluating the effects of these modalities used to the baby and mother during and after pregnancy.

These findings can be helpful to the government or other agencies involved in policy making to ensure a safe use of T\&CM among Malaysian women.

\section{References:}

1. Zhang X (2002) Traditional Medicine Strategy 2002-2005. World Health Organisation, Geneva, pp: 1-74.

2. http://tcm.moh.gov.my/v4/modules/mastop_publish/?tac=15

3. http://www.therakyatpost.com/life/wellness/

4. Hall HG, Griffiths DL, McKenna LG (2011) The use of complementary and alternative medicine by pregnant women: A literature review. Midwifery 27: $817-824$ 
Citation: Shaukat AR, Gnanasan S, Farooqui M (2016) The Status Quo and Future of Traditional and Complementary Medicine (T\&CM) Used During Pregnancy and Postpartum Period among Malaysian Women. Altern Integr Med 5: e120. doi:10.4172/2327-5162.1000e120

Page 2 of 2

5. Rahman AA, Sulaiman SA, Ahmad Z, Daud WN, Hamid AM, et al. (2008) Prevalance and pattern of use of herbal medicines during pregnancy in

6. http://www.babycentre.co.uk/a536346/herbal-remedies-in-pregnancy Tumpat District, Kelantan. Malays J Med Sci 15: 40-48. 